\title{
Esquema de Diversidade Cooperativa com Duas Estações Relay e Detecção Linear Coerente
}

\author{
Renato Machado, Robson Domingos Vieira e Mário de Noronha Neto
}

\begin{abstract}
Resumo-Neste artigo, apresenta-se um novo esquema de diversidade cooperativa que utiliza duas estações relay, um processo de detecção linear coerente no nó destino e modulação PSK. Neste esquema, os nós transmissor e receptor são equipados com uma única antena e as estações relay com duas. As estações relay utilizam uma pré-codificação linear para transmitir os dados detectados ao nó destino. Através de um canal de realimentação limitado, uma das estações relay utiliza a informação do estado do canal para multiplicar o símbolo detectado por um fator de correção antes de transmiti-lo ao nó destino. $O$ desempenho do esquema proposto é verificado através de uma comparação com a técnica MRC. Outras simulações analisando a influência do número de bits utilizados no canal de realimentação e a variação do valor da SNR entre o nó transmissor e as estações relay também são apresentadas.
\end{abstract}

Palavras-Chave-Canal de realimentação limitado, códigos espácio-temporais, detecção linear coerente, estações relay, diversidade na transmissão/recepção, sistemas MIMO.

Abstract - In this paper, we propose a new cooperative diversity scheme with two relay stations and linear coherent detection at the destination node and PSK modulation. In the proposed scheme, the transmit and receive nodes are equipped with a single antenna and the relay stations with two antennas. A linear precoding scheme is applied in the relay stations that transmit the detected symbol to the destination node. A limited feedback channel between one of the relays and the destination node is considered. This relay station, using the feedback CSI, multiply the detected symbol with a correction factor and then send it to the destination node which performs a linear coherent detection. The performance of the proposed scheme is compared with the well known MRC receiver technique with four receive antennas. In addition, the influence of the number of feedback bits and the SNR between the transmit node and relay stations are also addressed.

Keywords - Linear coherent detection, limited feedback channel, MIMO systems, relay stations, receive/transmit diversity, space-time codes.

\section{INTRODUÇÃO}

Atualmente, pode-se encontrar redes de comunicações sem fio que fornecem serviços de voz com alta qualidade, altas taxas de transmissão e serviços de multimídia. Porém, os sistemas de comunicações sem fio continuam em constante desenvolvimento já que novas aplicações e serviços mais elaborados e específicos passaram a ser requeridos. Um exemplo

R. Machado está com o Departamento de Engenharia Elétrica, Universidade Federal de Juiz de Fora, Juiz de Fora, MG, 36036-330, BRASIL. E-mail: renatomachado@engenharia.ufjf.br. R. D. Vieira está com Instituto Nokia de Tecnologia, Brasília, DF 70397-900, BRASIL. E-mail: robson.domingos@indt.org.br. M. Noronha-Neto está com o Instituto Federal de Santa Catarina, São José, SC 88130310, BRASIL. E-mail: noronha@ifsc.edu.br. claro dessa evolução são as novas especificações de requisitos que a ITU (international telecommunications union) está trabalhando para as futuras redes de comunicações sem fio, chamado de IMT-A (international mobile telecommunications - advanced) [1]. Taxas de dados na ordem de $1 \mathrm{G}$ bits/s e largura de banda de $100 \mathrm{MHz}$ são requisitos que distinguem os sistemas IMT-A dos sistemas $3 \mathrm{G}$ e das redes WiMAX (worldwide interoperability for microwave access) (802.16e). Assim, com o objetivo de atingir os requisitos determinados pelo ITU para futuras rede de comunicações sem fio, algumas tecnologias chaves devem ser estudadas e avaliadas. Dentre elas podemos destacar os sistemas MIMO (multiple-input multiple-output) com oito antenas, relay e o agrupamento de banda [2].

Os sistemas IMT-A utilizarão a técnica de acesso múltiplo OFDMA (orthogonal frequency-division multiple access) que facilita a multiplexação do canal de rádio nos domínios da frequência, do tempo e do espaço, facilitando, desse modo, o desenvolvimento de estratégias de alocação de recursos que explorem esses domínios. Atualmente as tecnologias 3G-LTE (long term evolution) [3] e o WiMAX [4], já contemplam este tipo de multiplexação. A utilização de múltiplas antenas na transmissão e na recepção já é mandatória nas tecnologias $3 \mathrm{G}$ LTE e WiMAX. Porém, um número limitado de quatro antenas para a transmissão e recepção foi adotado. Diversos estudos vem sendo realizados para avaliar a aplicação do relay nas redes de comunicações sem fio. Podemos citar, por exemplo, dois grupos de trabalhos no WiMAX (802.16j e 802.16m) que estão estudando a integração do conceito de relay no padrão [5]-[7].

Existem diversas estratégias de relay que podem explorar os benefícios da diversidade espacial obtidos da cooperação, tais como a amplifica e envia, demodula e envia e decodifica e envia. Na técnica amplifica e envia, as relays amplificam o sinal recebido e enviam o sinal amplificado para o destino [8], [9]. Na demodula e envia, a relay demodula individualmente cada símbolo, modula (ou não) o símbolo novamente e o envia para o destino [8]. Na técnica decodifica e envia, cada relay decodifica a mensagem recebida, codifica novamente (ou não) e envia a sequência codificada para o destino [9]-[11]. Em todas estratégias supramencionadas a diversidade espacial pode ser obtida combinando as múltiplas réplicas do sinal recebido oriundas do nó fonte e das relays.

Neste artigo, apresenta-se um novo esquema de diversidade cooperativa que utiliza duas estações relay e detecção linear coerente no nó destino. O esquema proposto considera sistemas com apenas uma única antena em cada nó transmissor e receptor e duas antenas nas estações relay. As estações relay 
utilizam uma pré-codificação linear para transmitir os dados detectados ao nó destino. Para realizar a detecção coerente o sistema utiliza um canal de realimentação limitado, consistindo em apenas alguns poucos bits de realimentação, utilizado para enviar informação sobre o estado do canal para uma das estações relay. Esta, por sua vez, multiplica o símbolo detectado por um fator de correção antes de transmiti-lo ao nó destino. Embora o esquema proposto seja apresentado apenas para sistemas que possuem uma única antena em cada nó transmissor e receptor e duas antenas nas estações relay, a generalização deste esquema já está sendo investigada.

$\mathrm{O}$ restante do artigo está organizado da seguinte maneira. $\mathrm{Na}$ próxima seção, apresenta-se o modelo do sistema. O esquema de diversidade cooperativa proposto é descrito na Seção III. $\mathrm{Na}$ Seção IV, apresentam-se os resultados de simulações. Finalmente, na Seção V, apresentam-se as conclusões e alguns comentários finais.

\section{Modelo do Sistema}

O modelo do sistema de comunicação sem fio cooperativo considerado nesta seção é composto por um nó transmissor, duas estações relay e um nó destino, como pode ser observado na Figura 1. O desvanecimento considerado no canal de comunicação sem fio é do tipo Rayleigh e os sinais recebidos nas estações relay e no nó destino são corrompidos pelo ruído Gaussiano. Os ganhos do canal $h_{1, i}^{1}$ e $h_{1, j}^{2}$, associados ao canal entre o nó 1 e as antenas $i$ e $j$ das estações relay 1 e 2 , respectivamente, bem como os ganhos $g_{i, 0}^{1}$ e $g_{j, 0}^{2}$, associados ao canal entre as antenas $i$ e $j$ das estações relay 1 e 2 e o nó 0 , respectivamente, para $i=1, \ldots, M_{1}, j=1, \ldots, M_{2}$, são modelados como variáveis aleatórias Gaussianas complexas independentes, circularmente simétricas, com média zero e variância $1 / 2$ por dimensão. Estes ganhos são considerados constantes durante um período de símbolo.

A cada instante de tempo $t$, o sinal $s_{t}$, pertencente a uma dada constelação, é transmitido do nó transmissor para as estações relay 1 e 2 , denotadas por $R_{1}$ e $R_{2}$, respectivamente.

$\mathrm{O}$ sinal $y_{t}^{l}$, recebido no instante de tempo $t$ pela $l$-ésima estação relay, é dado por:

$$
y_{t}^{l}=\sum_{i=1}^{M_{l}} \sqrt{P} h_{1, i}^{l} s_{t}+\eta_{1, i, t}^{l} \quad l=1,2 .
$$

em que $P$ é a potência total transmitida por cada estação relay em cada instante de tempo, e $\eta_{l, t}$ representa o ruído Gaussiano com média zero e variância $N_{0} / 2$ por dimensão. Assumindose que as estações relay 1 e 2 conhecem perfeitamente o canal, estas realizam a combinação de máxima razão (MRC, maximum ratio combining) seguindo com a detecção de máxima verossimilhança (MLD, maximum likelihood decoding), computando a seguinte métrica de decisão

$$
\left|y_{t}^{l}-\sum_{i=1}^{M_{l}} \sqrt{P} h_{1, i}^{l} s_{t}\right|^{2}
$$

A decisão é feita em favor do símbolo que resulta em uma menor métrica.

No instante de tempo $t+1$, as estações relay 1 e 2 encaminham os sinais detectados, $\hat{s}_{t}^{1}$ e $\hat{s}_{t}^{2}$, simultaneamente ao nó destino, chamado por nó 0 . As estações relay 1 e 2 conhecem os ganhos do canal entre $R_{1}$ e o nó 0 e entre $R_{2}$ e o nó 0, e realizam uma formatação de feixe ótima [12]. Como a potência transmitida total por estação relay em cada instante de tempo $t$ é $P$, a potência transmitida por cada antena nas estações relay são normalizadas para ser $P / M_{1}$ e $P / M_{2}$.

O sinal $y_{t+1}^{0}$, recebido no instante de tempo $t+1$ pelo nó destino, é dado por:

$y_{t+1}^{0}=\sum_{i=1}^{M_{1}} \sqrt{P / M_{1}} w_{i}^{1} g_{i, 0}^{1} \hat{s}_{t}^{1}+\sum_{j=1}^{M_{2}} \sqrt{P / M_{2}} w_{j}^{2} g_{j, 0}^{2} \hat{s}_{t}^{2}+\eta_{0, t+1}$

em que $w_{i}^{1}$ e $w_{j}^{2}$ são sinais formatados, e $\eta_{0, t+1}$ representa o ruído Gaussiano branco complexo com média zero e variância $N_{0} / 2$ por dimensão complexa. Assumindo-se estimação perfeita do canal no nó destino, a detecção ML pode ser realizada computando a métrica de decisão

$$
\left|y_{t+1}^{0}-\sum_{i=1}^{M_{1}} \sqrt{P / M_{1}} w_{i} g_{i, 0}^{1} s_{t}-\sum_{i=j}^{M_{2}} \sqrt{P / M_{2}} w_{j} g_{j, 0}^{2} s_{t}\right|^{2}
$$

A decisão é feita em favor do símbolo que resulta em uma menor métrica.

\section{ESQUema DE DIVERSIDAdE COOPERATIVA COM Duas Estações Relay e Detecção Linear Coerente}

Nesta seção, o esquema de diversidade cooperativa é apresentado. Assume-se que a CSI de $h_{1, i}^{1}$ e $h_{1, j}^{2}$ estão disponíveis nas estações relay 1 e 2 , e que a CSI de $g_{i, 0}^{1}$ e $g_{j, 0}^{2}, i=$ $1, \ldots, M_{1}, j=1, \ldots, M_{2}$, sejam perfeitamente conhecidas no nó destino e nas estações relay 1 e 2 . Há um canal de realimentação disponível entre o nó destino e a estação relay 2 (ou a estação relay 1), através do qual o nó 0 envia informação sobre o estado do canal para $R_{2}$ (ou $R_{1}$ ), permitindo que esta transmita o símbolo $\hat{s}_{t}^{2}$ de uma maneira mais apropriada para se aumentar o ganho de SNR no nó destino.

Daqui por diante, o índice $t$ será omitido com o propósito de simplificar a notação das próximas equações.

\section{A. Esquema de Transmissão}

No esquema proposto, o nó 1 transmite seu próprio símbolo de informação no primeiro período de transmissão para as estações relay 1 e 2 que detectam esse símbolo e retransmitemno, utilizando a técnica de formatação de feixe ótima, para o nó destino. Por simplicidade, assume-se que as estações relay têm apenas duas antenas com alocação uniforme de potência, sendo a potência total dada por $P=1 .^{1}$

Seja $s_{1}$ o símbolo transmitido pelo nó 1 , pertencente a uma constelação de símbolos $M$-PSK, ou seja, com energia média unitária. Os sinais recebidos em banda base nas estações relay 1 e 2 são dados por

$$
\begin{aligned}
& y^{1}=h_{1,1}^{1} s_{1}+h_{1,2}^{1} s_{1}+\eta_{1,1}^{1}+\eta_{1,2}^{1} \\
& y^{2}=h_{1,1}^{2} s_{1}+h_{1,2}^{2} s_{1}+\eta_{1,1}^{2}+\eta_{1,2}^{2}
\end{aligned}
$$

\footnotetext{
${ }^{1}$ Duas antenas por estação relay e alocação uniforme de potência não são restrições mandatórias para o esquema de transmissão proposto.
} 


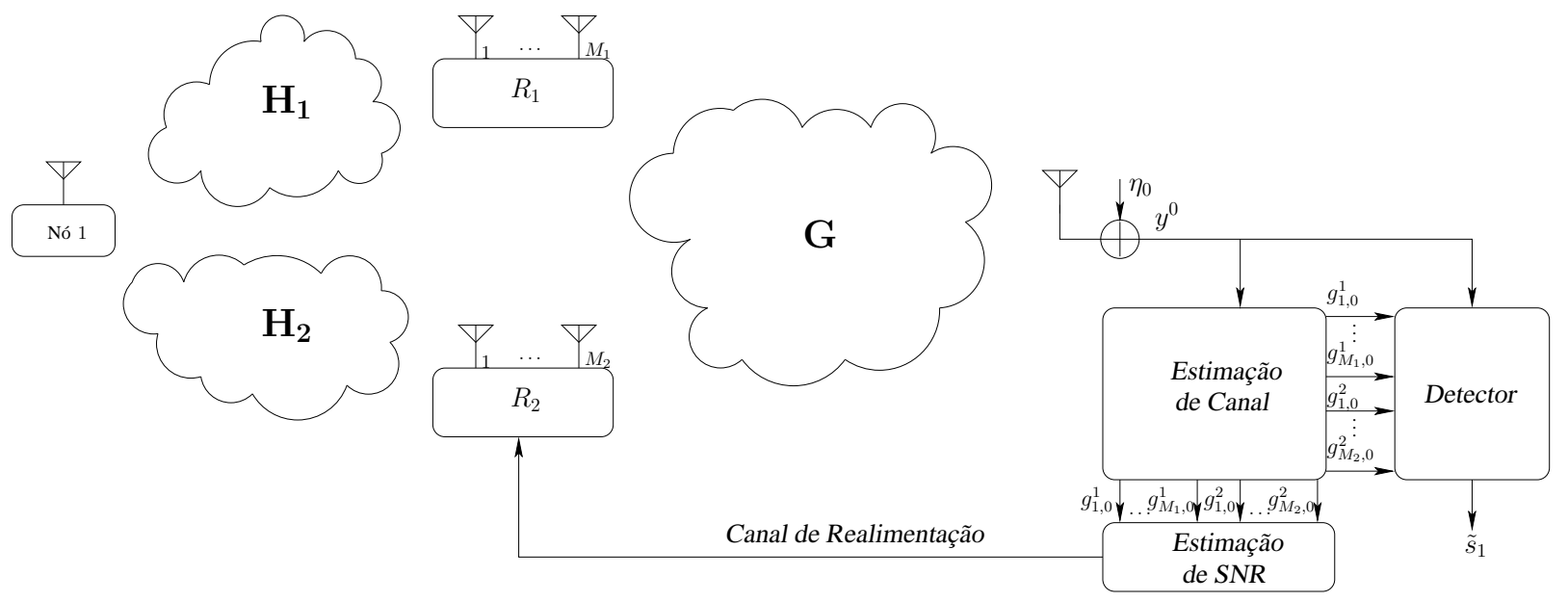

Fig. 1. Diagrama de bloco do modelo do sistema.

respectivamente, em que $\eta_{1}$ e $\eta_{2}$ representam os ruídos AWGN.

Considerando-se que o conhecimento perfeito do estado do canal está disponível nas estações relay, então a deteç̧ão ML pode ser realizada da seguinte forma:

$$
\hat{s}_{1}^{1}=\arg \min _{s}\left|y^{1}-h_{1,1}^{1} s_{1}-h_{1,2}^{1} s_{1}\right|^{2}
$$

e

$$
\hat{s}_{1}^{2}=\arg \min _{s}\left|y^{2}-h_{1,1}^{2} s_{1}-h_{1,2}^{2} s_{1}\right|^{2}
$$

No segundo período de transmissão, $R_{1}$ e $R_{2}$ "encaminham" os símbolos detectados $\hat{s}_{1}^{1}$ e $\hat{s}_{1}^{2}$ simultaneamente para o nó destino. Os sinais são ponderados de maneira apropriada, tal que a transmissão por formatação de feixe ótima possa ser empregada em cada uma das estações relay.

\section{B. Detecção Linear Coerente}

O sinal recebido no nó destino pode ser visto como combinação dos dois vetores representados na Figura 2, e é dado por

$$
\begin{aligned}
y_{D}= & \sqrt{P / 2} w_{1}^{1} g_{1,0}^{1} \hat{s}_{1}^{1}+\sqrt{P / 2} w_{2}^{1} g_{2,0}^{1} \hat{s}_{1}^{1}+ \\
& \exp \left\{j \theta_{d}\right\}\left(\sqrt{P / 2} w_{1}^{2} g_{1,0}^{2} \hat{s}_{1}^{2}+\sqrt{P / 2} w_{2}^{2} g_{2,0}^{2} \hat{s}_{1}^{2}\right)+\eta_{D}
\end{aligned}
$$

em que $\eta_{D}$ representa o termo AWGN, $w_{i}^{1}=\exp \left\{j \theta_{i}^{1}\right\}, w_{j}^{2}=$ $\exp \left\{j \theta_{j}^{2}\right\}, i, j=1,2$, são os pesos de cada sinal, e $\theta_{d}$ é o fator de correção cuja escolha é baseada nos bits de informação que a estação relay 2 recebe do nó destino através do canal de realimentação. Assumindo $P=1$ e $w_{1}^{1}=w_{1}^{2}=\exp \{j 0\}$, pode-se reescrever (8) como

$$
\begin{aligned}
y_{D}= & \sqrt{1 / 2} g_{1,0}^{1} \hat{s}_{1}^{1}+\sqrt{1 / 2} w_{2}^{1} g_{2,0}^{1} \hat{s}_{1}^{1}+ \\
& \exp \left\{j \theta_{d}\right\}\left(\sqrt{1 / 2} g_{1,0}^{2} \hat{s}_{1}^{2}+\sqrt{1 / 2} w_{2}^{2} g_{2,0}^{2} \hat{s}_{1}^{2}\right)+\eta_{D}
\end{aligned}
$$

$\mathrm{O}$ processamento linear seguinte, considerando que os símbolos transmitidos pertençam a uma constelação $M$ PSK, produz a entrada desejada para o detector de maxima

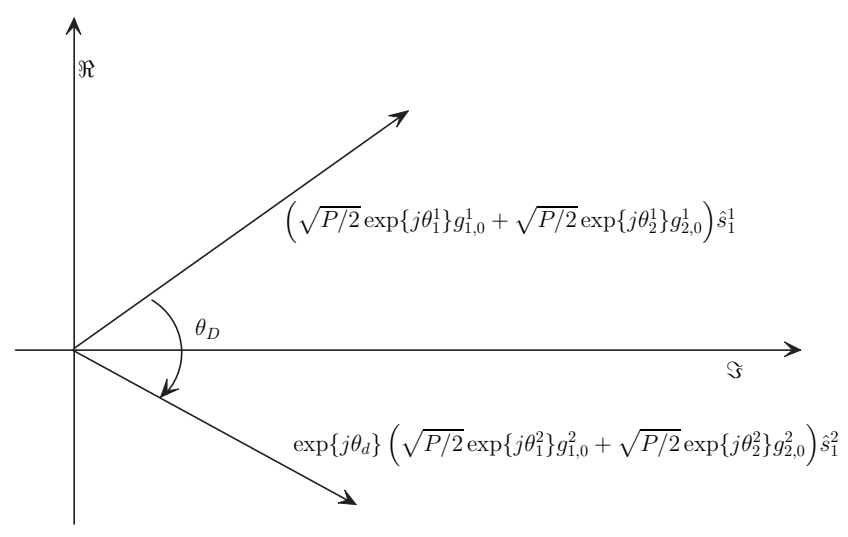

Fig. 2. Sinal recebido no nó destino.

verossimilhança:

$$
\begin{gathered}
\tilde{s}_{1}=y_{D}\left(g_{1,0}^{1}+\exp \left\{j \theta_{2}^{1}\right\} g_{2,0}^{1}+\right. \\
\left.\left(g_{1,0}^{2}+\exp \left\{j \theta_{2}^{2}\right\} g_{2,0}^{2}\right) \exp \left\{j \theta_{d}\right\}\right)^{*} \\
=\quad\left(\left|g_{1,0}^{1}\right|^{2}+\left|g_{2,0}^{1}\right|^{2}+\beta_{1}\right) \hat{s}_{1}^{1} / \sqrt{2}+\left(\left|g_{1,0}^{2}\right|^{2}+\left|g_{2,0}^{2}\right|^{2}+\right. \\
\left.\beta_{2}\right) \hat{s}_{1}^{2} / \sqrt{2}+\eta_{D}\left(g_{1,0}^{1}+\exp \left\{j \theta_{2}^{1}\right\} g_{2,0}^{1}+\left(g_{1,0}^{2}+\right.\right. \\
\left.\left.\quad \exp \left\{j \theta_{2}^{2}\right\} g_{2,0}^{2}\right) \exp \left\{j \theta_{d}\right\}\right)^{*}
\end{gathered}
$$

em que “*” denota conjugado complexo,

$$
\begin{aligned}
\beta_{1}= & 2 \Re\left\{g_{1,0}^{1}\left(g_{2,0}^{1} \exp \left\{\theta_{2}^{1}\right\}\right)^{*}\right\}+g_{1,0}^{1}\left(g_{1,0}^{2} \exp \left\{j \theta_{d}\right\}\right)^{*}+ \\
& g_{2,0}^{1} \exp \left\{\theta_{2}^{1}\right\}\left(g_{1,0}^{2} \exp \left\{j \theta_{d}\right\}\right)^{*}+g_{1,0}^{1}\left(g_{2,0}^{2} \exp \left\{j \theta_{2}^{2}\right\}\right. \\
& \left.\exp \left\{j \theta_{d}\right\}\right)^{*}+g_{2,0}^{1} \exp \left\{\theta_{2}^{1}\right\}\left(g_{2,0}^{2} \exp \left\{j \theta_{2}^{2}\right\} \exp \left\{j \theta_{d}\right\}\right)^{*}
\end{aligned}
$$

e

$$
\begin{aligned}
\beta_{2}= & 2 \Re\left\{g_{1,0}^{2}\left(g_{2,0}^{2} \exp \left\{j \theta_{2}^{2}\right\} \exp \left\{j \theta_{d}\right\}\right)^{*}\right\}+g_{1,0}^{1 *} g_{1,0}^{2} \\
& \exp \left\{j \theta_{d}\right\}+g_{1,0}^{2} \exp \left\{j \theta_{d}\right\}\left(g_{2,0}^{1} \exp \left\{j \theta_{2}^{1}\right\}\right)^{*}+g_{2,0}^{2} \\
& \exp \left\{j \theta_{2}^{2}\right\} \exp \left\{\theta_{d}\right\} g_{1,0}^{1 *}+g_{2,0}^{2} \exp \left\{j \theta_{2}^{2}\right\} \exp \left\{j \theta_{d}\right\} g_{2,0}^{1 *}
\end{aligned}
$$

Se for assumido que $\hat{s}_{1}^{1}=\hat{s}_{1}^{2}=s_{1}$, então pode-se reescrever 
(10) como

$$
\begin{aligned}
\tilde{s}_{1}= & \left(\left|g_{1,0}^{1}\right|^{2}+\left|g_{2,0}^{1}\right|^{2}+\left|g_{1,0}^{2}\right|^{2}+\left|g_{2,0}^{2}\right|^{2}+\beta\right) s_{1} / \sqrt{2}+ \\
& \eta_{D}\left(g_{1,0}^{1}+\exp \left\{j \theta_{2}^{1}\right\} g_{2,0}^{1}+\left(g_{1,0}^{2} \exp \left\{j \theta_{2}^{2}\right\} g_{2,0}^{2}\right)\right. \\
& \left.\exp \left\{j \theta_{d}\right\}\right)^{*}
\end{aligned}
$$

em que

$$
\begin{aligned}
\beta= & \beta_{1}+\beta_{2} \\
= & 2 \Re\left\{g_{1,0}^{1}\left(g_{2,0}^{1} \exp \left\{\theta_{2}^{1}\right\}\right)^{*}\right\}+ \\
& 2 \Re\left\{g_{1,0}^{1}\left(g_{1,0}^{2} \exp \left\{j \theta_{d}\right\}\right)^{*}\right\}+ \\
& 2 \Re\left\{g_{1,0}^{2}\left(g_{2,0}^{2} \exp \left\{j \theta_{2}^{2}\right\} \exp \left\{j \theta_{d}\right\}\right)^{*}\right\}+ \\
& 2 \Re\left\{g_{2,0}^{1} \exp \left\{\theta_{2}^{1}\right\}\left(g_{2,0}^{2} \exp \left\{j \theta_{2}^{2}\right\} \exp \left\{j \theta_{d}\right\}\right)^{*}\right\}
\end{aligned}
$$

O sinal $s_{1}$ tem energia média unitária, portanto a razão sinalruído (SNR) do sinal recebido (8) é

$$
\gamma=\frac{\left|g_{1,0}^{1}\right|^{2}+\left|g_{2,0}^{1}\right|^{2}+\left|g_{1,0}^{2}\right|^{2}+\left|g_{2,0}^{2}\right|^{2}+\beta}{2} \gamma_{0}
$$

em que $\gamma_{0}=1 / N_{0}$. Como $\beta$ pode assumir valores negativos quando um canal de realimentação não é considerado, então a ordem de diversidade completa não pode ser garantida.

No esquema proposto, assume-se que existe um canal de realimentação livre de erros e de atrasos, através do qual $b_{f}$ bits podem ser enviados do nó 0 para a estação relay 2 . Baseado nas condições instantâneas do canal, o nó 0 determina qual $\theta_{d}, \theta_{d} \in 2^{b_{f}}$-PSK, retorna a máxima SNR instantânea. Em outras palavras, o nó 0 determina qual $\theta_{d}$ resulta no maior valor de $\beta$.

Quando um canal de realimentação é considerado, mesmo que somente um único bit de realimentação seja permitido, $\theta_{d}$ pode ser escolhido de tal maneira que $\beta$ seja um número positivo. Logo, se o canal de realimentação for utilizado de maneira apropriada, está claro em (11) que a diversidade espacial de ordem 4 é alcançada no nó destino.

\section{Resultados de Simulação}

Nesta seção, apresentam-se resultados de simulação para mostrar o desempenho de erro do esquema de comunicação cooperativo descrito na Seção III. Os resultados são expressos em termos da taxa de erro de bit (BER) versus a razão sinal ruído média entre o nó 1 e as estações relay $\left(\mathrm{SNR}_{0}\right)$, ou a razão sinal ruído média entre as estações relay e o nó destino $\left(\mathrm{SNR}_{1}\right)$. A modulação BPSK é a constelação considerada em todas as simulações apresentadas nesta seção. A BER foi estimada baseada na contagem de pelo menos 400 bits errados para cada valor de $\mathrm{SNR}_{0}$ ou $\mathrm{SNR}_{1}$. O canal é considerado plano e quase-estático, ou seja, o canal é constante durante a duração de um bloco de transmissão e varia aleatoriamente de um bloco de transmissão para outro. Assume-se que os ganhos dos canais são amostras independentes de um processo aleatório Gaussiano complexo com média zero e variância 1/2 por dimensão.

A Figura 3 mostra o desempenho do esquema proposto quando o valor de $\mathrm{SNR}_{0}$ é 5,15 , ou $25 \mathrm{~dB}$ maior do que o valor de $\mathrm{SNR}_{1}$. O número de bits de realimentação do nó destino para estação relay 2 é igual a $3\left(b_{f}=3\right)$. A Figura 3 também revela que o desempenho do sistema está associado

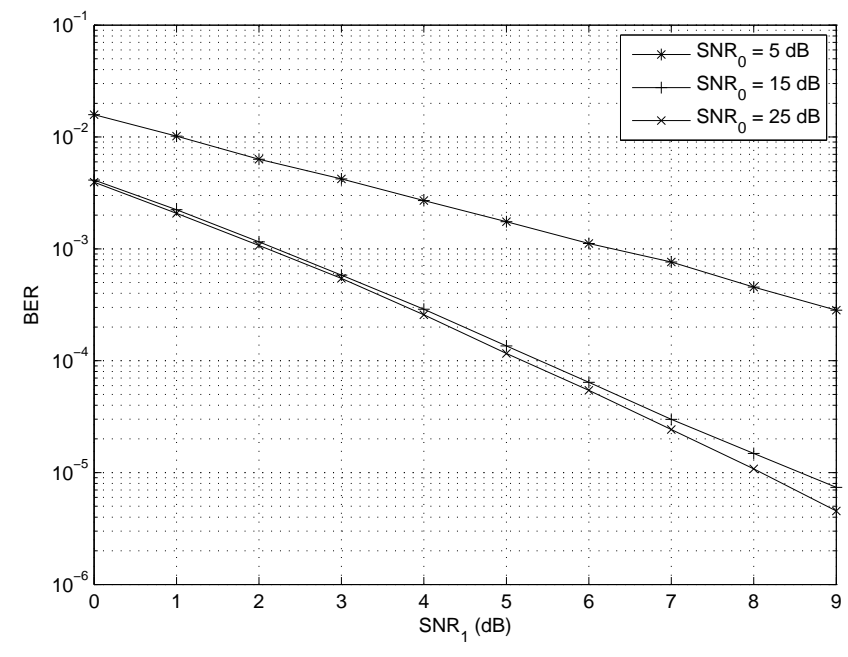

Fig. 3. Desempenho de erro do esquema proposto para diferentes valores de $\mathrm{SNR}_{0}$ e $b_{f}=3$.

ao valor atribuído a $\mathrm{SNR}_{0}$. Pode-se notar que há perda de desempenho conforme o valor de $\mathrm{SNR}_{0}$ decresce. Esta perda de desempenho pode ser explicada pelo fato de que para baixos valores de $\mathrm{SNR}_{0}$ as estações relay detectam e retransmitemo (propagam) mais símbolos errados do que em condições de $\mathrm{SNR}_{0}$ mais elevadas.

Para se verificar qual o valor mínimo de $\mathrm{SNR}_{0}$ que garante a diversidade completa para o sistema, fixou-se alguns valores de $\operatorname{SNR}_{1}(1,3$, e $5 \mathrm{~dB})$, fez-se $b_{f}=3$, e variou-se o valor de $\mathrm{SNR}_{0}$ de 0 a $30 \mathrm{~dB}$. A Figura 4 apresenta esse resultado. Nessa figura, observa-se que quando $\mathrm{SNR}_{0}$ é aproximadamente 20 $\mathrm{dB}$ maior do que a $\mathrm{SNR}_{1}$ o desempenho do sistema satura. A partir desta observação pode-se concluir que existe uma restrição de valores entre $\mathrm{SNR}_{0}$ e $\mathrm{SNR}_{1}$ que se respeitada permite ao sistema alcançar diversidade completa. A Figura 4 também apresenta um limitante inferior para cada valor de $\mathrm{SNR}_{1}$ para evidenciar a saturação da BER. Os limitantes inferiores foram obtidos considerando-se uma transmissão sem ruído (AWGN) entre o nó fonte e as estações relay. Para mostrar a perda de desempenho quando a restrição de relação entre $\mathrm{SNR}_{0}$ e $\mathrm{SNR}_{1}$ não é mantida, a Figura 5 é apresentada. Nesta figura, para cada valor fixo de $\mathrm{SNR}_{0}\left(\mathrm{SNR}_{0}=15,20\right.$, ou $25 \mathrm{~dB}$ ), o valor de $\mathrm{SNR}_{1}$ varia de 0 a $20 \mathrm{~dB}$.

Na Figura 6 é possível se observar o influência do número de bits de realimentação no desempenho do sistema. Conforme apresentado nesta figura, o desempenho de erro é muito próximo do limitante inferior quando $b_{f}=3$ bits, indicando que praticamente não há mais ganho de desempenho de erro quando o nó destino envia mais do que 3 bits de realimentação para a estação relay 2. O valor adotado para $\mathrm{SNR}_{0}$ foi de 25 $\mathrm{dB}$.

Na Figura 7, mostra-se o desempenho do esquema proposto operando em $\mathrm{SNR}_{0}=25 \mathrm{~dB}$ e três bits de realimentação. Com o propósito de comparação e para se visualizar que o esquema cooperativo proposto pode alcançar diversidade completa na recepção, apresenta-se na mesma figura a curva do esquema MRC com quatro antenas receptoras (sistema com grau de 


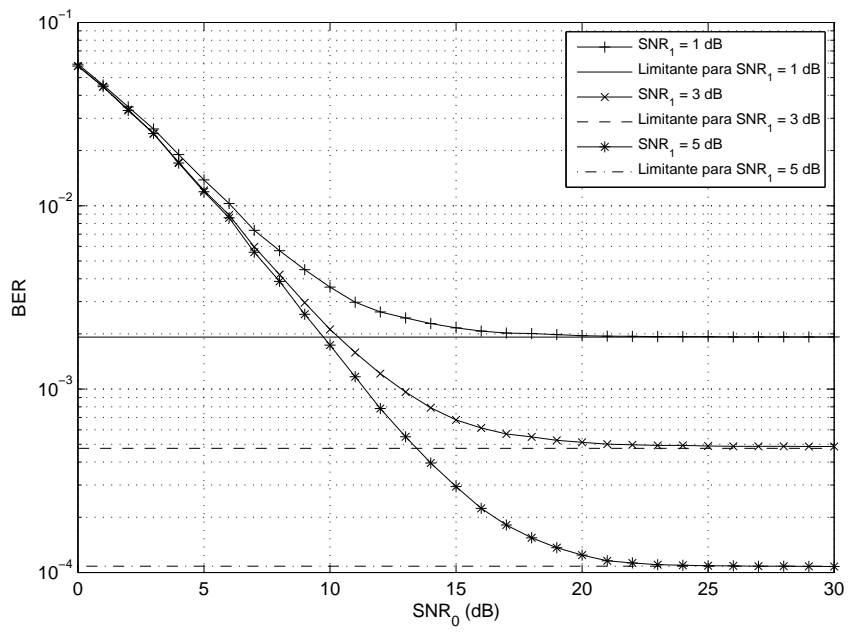

Fig. 4. Efeito da variação de $\mathrm{SNR}_{0}$ no desempenho do sistema para diferentes valores de $\mathrm{SNR}_{1}$.

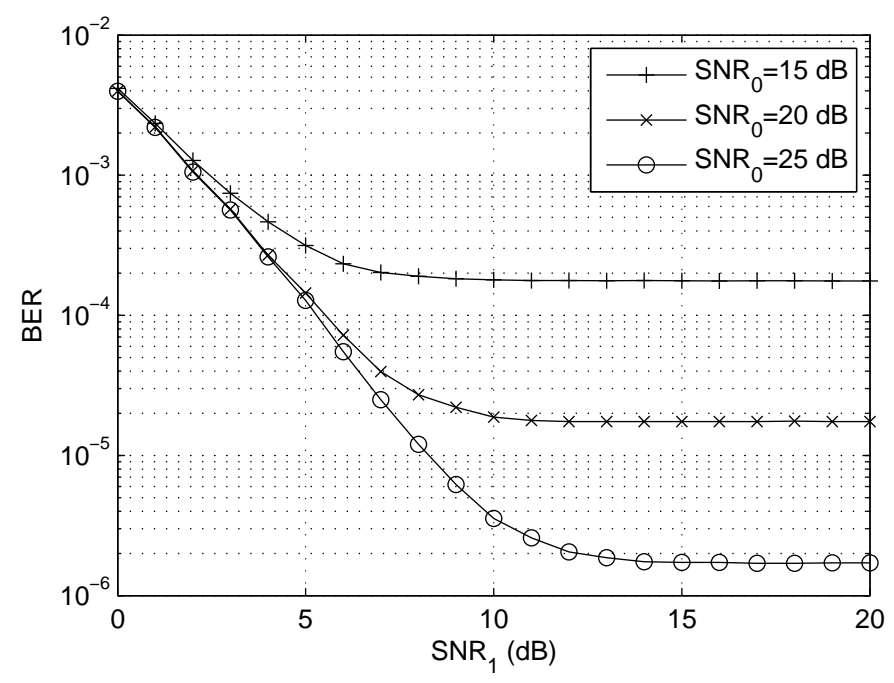

Fig. 5. Desempenho do esquema proposto para diferentes valores de $\mathrm{SNR}_{0}$.

diversidade espacial igual a 4). A partir deste resultado, já bastante conhecido da literatura, fica visivelmente claro que o esquema proposto apresenta diversidade completa, ou seja, de ordem 4. Ainda nessa figura, pode-se notar que o esquema de diversidade cooperativa apresenta um ganho de SNR de aproximadamente $2 \mathrm{~dB}$ sobre o esquema MRC. Esse ganho de SNR ocorre basicamente em função de dois fatores: i) os bits de realimentação fornecem informação necessária para que $\beta$ em (11) seja sempre um número real positivo; ii) a potência de transmissão em cada uma das antenas nas estações relay 1 e 2 , é normalizada $\left(P / M_{1}\right.$ e $\left.P / M_{2}\right)$, totalizando uma potência de transmissão $P=1$ em cada uma das estações relay, enquanto que no esquema MRC, que tem apenas uma única antena transmissora, a potência de transmissão total normalizada é $P=1$.

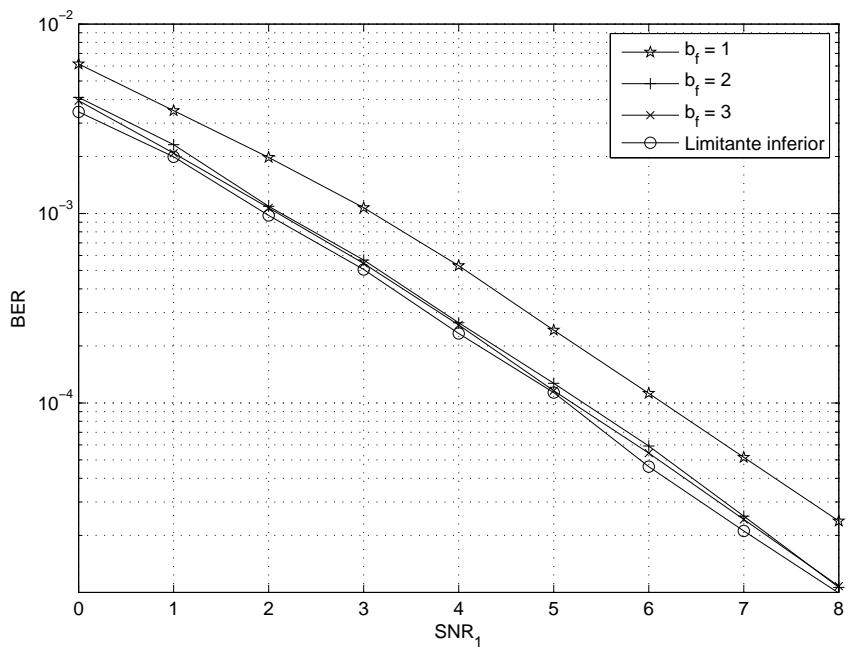

Fig. 6. Desempenho do esquema proposto para diferentes valores de $b_{f}$.

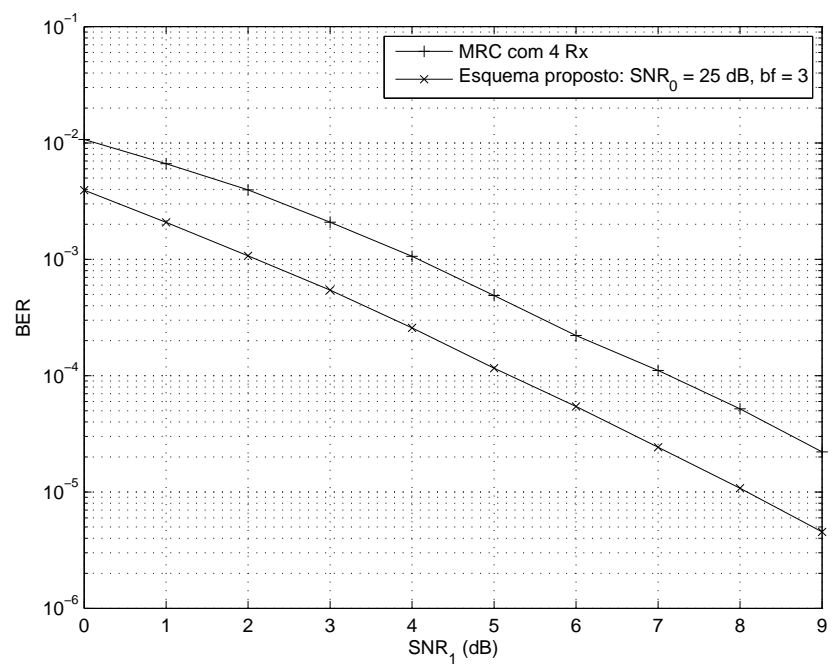

Fig. 7. Comparação de desempenho: Esquema proposto versus MRC.

\section{Conclusões e Comentários Finais}

Neste artigo, propôs-se um novo esquema de diversidade cooperativa com duas estações relay e detecção linear coerente no nó destino. Nesse esquema, um canal de realimentação limitado está disponível entre o nó destino e a estação relay 2. Mostrou-se que quando a estação relay 2 utiliza apropriadamente a informação do estado do canal recebida através do canal de realimentação, então a diversidade espacial completa pode ser obtida no nó destino através do esquema proposto. Para cada valor de $\mathrm{SNR}_{1}$ existe um valor mínimo correspondente para $\mathrm{SNR}_{0}\left(\mathrm{SNR}_{0}>\mathrm{SNR}_{1}\right)$ que garante que a ordem de diversidade completa é obtida no nó destino e que acima deste valor a BER no nó destino não pode ser mais melhorada. Logo, há um problema de otimização em aberto que consiste em encontrar a melhor relação entre $\mathrm{SNR}_{0}$ e $\mathrm{SNR}_{1}$, tal que a ordem de diversidade completa no nó destino seja garantida. Embora todas simulações tenham sido realizadas considerando 
a modulação BPSK e apenas a presença de duas estações relay, cada uma delas com somente duas antenas transmissoras, a extensão deste trabalho para um cenário mais geral (mais do que duas antenas por estação relay, mais do que duas estações relay, estimação imperfeita do canal, constelações $M$-PSK ou $M$-QAM, etc.) está sob atual investigação.

\section{REFERÊNCIAS}

[1] ITU-R Draft New Recommendation, "Vision, framework and overall objectives of the future development of IMT-2000 and systems beyond IMT-2000."

[2] ITU-R Draft New Recommendation, "Guidelines for evaluation of radio interface technolgies for IMT-Adavanced."

[3] 3GPP TS 36.211 V8.3.0 (2008-05), "Evolved Universal Terrestrial Radio Access (E-UTRA), Physical Channels and Modulation (Release 8)."

[4] IEEE Std 802.16e-2005, "Air Interface for Fixed and Mobile Broadband Wireless Access Systems", Amendment 2: Physical and Medium Access Control Layers for Combined Fixed and Mobile Operation in Licensed Bands

[5] http://ieee802.org/16/
[6] IEEE 802.16 Broadband Wireless Access Working Group, "Draft IEEE 802.16m System Description Document", July 2008.

[7] Part 16: Air Interface for Fixed and Mobile Broadband Wireless Access Systems-Multihop Relay Specification, "IEEE P802.16j/D2", December 2007.

[8] D. Chen and J. N. Laneman, "Modulation and demodulation for cooperative diversity in wireless systems," IEEE Trans. Wireless Commun., vol. 5, pp. 1785-1794, July 2006.

[9] J. N. Laneman, D. N. C. Tse, and G. W. Wornell, "Cooperative diversity in wireless networks: Efficient protocols and outage behavior," IEEE Trans. Inf. Theory, vol. 50, pp. 3062-3080, December 2004.

[10] J. N. Laneman and G. W.Wornell, "Distributed space-time coded protocols for exploiting cooperative diversity in wireless networks," IEEE Trans. Inf. Theory, vol. 49, no. 10, pp. 2415-2425, October 2003.

[11] G. Kramer, M. Gastpar, and P. Gupta, "Capacity theorems for wireless relay channels," in Proc. Allerton Conf. Communications, Control, Computing, Monticello, IL, October, 2003, pp. 1074-1083.

[12] Khoshnevis, B., Wei Yu, and Adve, R., "Grassmannian beamforming for MIMO amplify-and-forward relaying," in Proc. 42nd Annual Conference on Information Sciences and Systems, 2008. CISS 2008, Princeton, NJ, March 2008, pp. 161-166. 